\title{
Estimating MCC System Dryness Index using the Vineyard Water Indicator
}

\author{
Marco Antônio Fonseca Conceição ${ }^{1}$, Reginaldo Teodoro de Souza ${ }^{1}$, and Jorge Tonietto ${ }^{2}$ \\ ${ }^{1}$ Embrapa Grape and Wine, Tropical Viticulture Experimental Station, Jales, SP, Brazil \\ ${ }^{2}$ Embrapa Grape and Wine, Bento Gonçalves, RS, Brazil
}

\begin{abstract}
The Dryness Index (DI) is one of the three Geoviticulture Multicriteria Climatic Classification System (MCC System) indices and its calculation is based on a soil water balance approach. However, other climatic indices can be used for the same purpose. One of them is the Vineyard Water Indicator (VWI) that represents the ratio between the total rainfall and the vineyard water requirement during the productive period of the culture. When compared to DI, the VWI presents a simpler calculation methodology. Therefore, the aim of the present study was to establish a model to estimate DI based on VWI values. Climate data of 80 winegrowing regions in 18 countries were used. Four regression models were evaluated: linear, quadratic, logarithmic and the Mitscherlich model. Real and simulated data were compared using the confidence coefficient (c) that corresponds to the product of the correlation coefficient ( $r$ ) by the exactness coefficient (d). The best fit was obtained employing the quadratic model and DI can be calculated using the following equation: $\mathrm{DI}=-363.84 \mathrm{VWI}^{2}+834.47 \mathrm{VWI}-257.17\left(\mathrm{R}^{2}=0.93\right)$, for $\mathrm{VHI}<0.905$. For VHI values equal to or greater than 0.905 , DI is constant and equal to 200 .
\end{abstract}

\section{Introduction}

Several climate classification systems have been employed to characterize grape-growing regions in many countries of the world. Some of them use only temperature data $[1,2]$ while others include evapotranspiration and rainfall information to evaluate water scarcity as well as plant health risks due to excessive rainfall $[3,4]$.

The Geoviticulture Multicriteria Climatic Classification System (MCC System) is an important tool for viticultural zoning studies and has been used in many countries for this purpose and for climate change impact evaluation [5-7]. The MCC System presents three indices, one of which is the Dryness Index (DI) that is based on a soil water balance (SWB) approach. The DI calculation needs a monthly algorithm procedure.

There are other water indices that could be used to characterize grape-growing regions based on a SWB approach, as the Thorthwaite and Mather index [3]. However, this approach requires monthly calculations and is dependent on soil water conditions. On the other hand, the Vineyard Water Indicator (VWI) can also be used for the same purpose but presents an easier calculation methodology [8]. It represents the ratio between the total rainfall and the vineyard water requirement during the productive period of the culture.

The aim of the present study was, therefore, to establish a model to estimate DI based on VWI values.

\section{Material and methods}

Climate data of 80 winegrowing regions in 18 countries were used [9]. The Dryness Index (DI) was calculated using the following expression [5]:

$$
D I=\sum(W o+P-T v-E s)
$$

where "Wo" is the initial useful soil water reserve, which can be accessed by the roots (mm); "P" is the monthly precipitation $(\mathrm{mm})$; "Tv" is the potential monthly transpiration of the vineyard (mm); "Es" is the monthly direct evaporation from the soil (mm). The calculation considered two different periods: from April to September for the North hemisphere; and from October to March for the South hemisphere.

The "Tv" was calculated using the expression:

$$
T v=E T P \cdot k
$$

where "ETP" is the potential evapotranspiration $(\mathrm{mm})$ calculated by the standard Penman-Monteith method [9]; $\mathrm{k}$ is the coefficient of radiation absorption by vine plant $(\mathrm{k}=0.1$ for the first month, 0.3 for the second month and 0.5 for the other months). "Es" was calculated using the expression:

$$
E s=(E T P / N) \cdot(1-k) \cdot J P m
$$

where $\mathrm{N}$ is the number of days in the month and JPm is the number of days of effective evaporation from soil per month, that was estimated dividing P per 5 and should be $\leq \mathrm{N}$. Table 1 shows the different climatic classes for DI.

The Vineyard Water Indicator (VWI) was calculated using the following equation [8]:

$$
V W I=P / E T P
$$

In the original work [8], instead of ETP, it was used the grapevine water demand or crop evapotranspiration (ETc), 
Table 1. Class, acronym and class interval for the Dryness Index (DI).

\begin{tabular}{|c|c|c|}
\hline Class & Acronym & Class interval $(\mathbf{m m})$ \\
\hline Humid & DI-2 & DI $>150$ \\
\hline Sub-humid & DI-1 & $150 \geq$ DI $>50$ \\
\hline Moderately dry & DI +1 & $50 \geq$ DI $>-100$ \\
\hline Very dry & DI +2 & $-100 \geq$ DI \\
\hline
\end{tabular}

Table 2. Correlation coefficient (r), exactness index (d), confidence index (c) and performance classification for the regression models.

\begin{tabular}{|c|c|c|c|c|}
\hline Model & r & d & c & Performance \\
\hline Logarithmic & 0,96 & 0,92 & 0,88 & Excellent \\
\hline Linear & 0,96 & 0,94 & 0,90 & Excellent \\
\hline Quadratic & 0,98 & 0,96 & 0,94 & Excellent \\
\hline Mitscherlich & 0,97 & 0,94 & 0,91 & Excellent \\
\hline
\end{tabular}

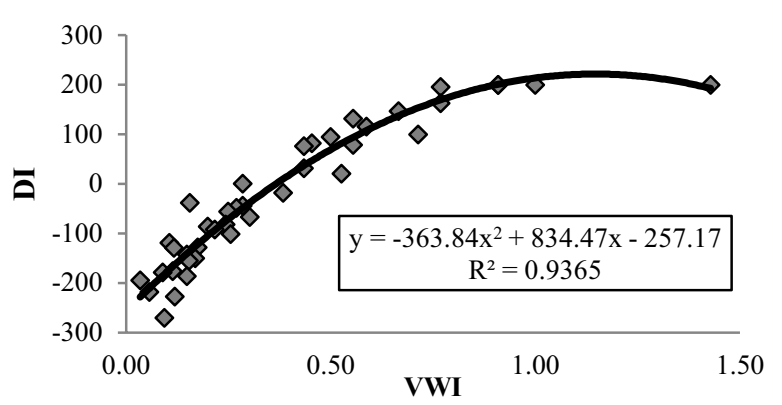

Figure 1. Quadratic regression model between VWI and DI values.

with the ETc calculated by multiplying the ETP by the crop coefficients $(\mathrm{Kc})$. The $\mathrm{Kc}$ values, however, varies depending on growing conditions, making it difficult to compare results between regions. Therefore, it was decided to consider a fixed $\mathrm{Kc}$ equal to 1.0 , which made the ETc becomes equal to ETP.

Forty winegrowing regions climate data were used for fit the regression models: linear, quadratic, logarithmic and the Mitscherlich model [10]. The model performances were evaluated using the confidence index (c) that corresponds to the product of the correlation coefficient (r) by the exactness index (d) [11]. The performance was classified as excellent for values higher than 0.85 ; very good between 0.76 and 0.85 ; good between 0.66 and 0.75 ; regular between 0.51 and 0.65 ; and bad for values lower than 0.50 . The other 40 regions data were employed to compare real and simulated data.

\section{Results and discussion}

All models presented excellent performances and the best confidence index (c) was obtained employing the quadratic one (Table 2).

The quadratic model equation is shown in Fig. 1. This equation is valid for of VWI values lower than 0.905 . For higher values, the DI becomes constant and equal to 200, which is the limit for DI values.

Real DI data were compared with DI simulated values calculated from VWI using Fig. 1 equation. The model underestimated the real DI values in 5\%, approximately (Fig. 2).

The excellent performance of the quadratic model (Table 2 and Fig. 1) means that VWI could be use in viticultural zoning and other studies, including climate change impact evaluation. In addition to an easier

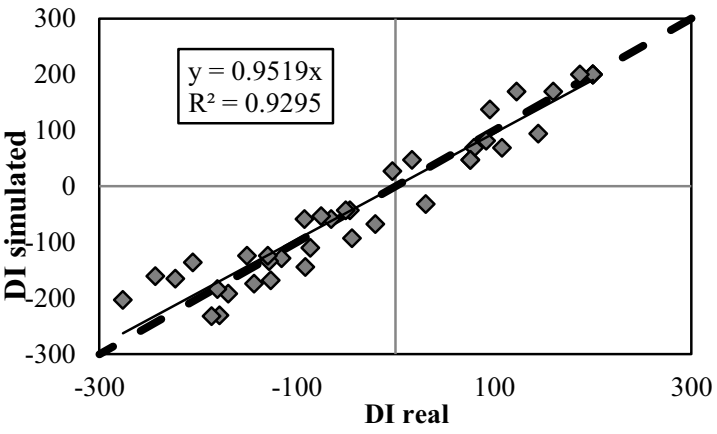

Figure 2. Comparing real and simulated DI values.

calculation procedure, the use of VWI does not require soil water information, since VWI is calculated dividing $\mathrm{P}$ by ETP. On the other hand, DI needs soil water information and employs a complex monthly algorithm to its calculation.

It should be noted, however, that despite the good agreement between real and simulated DI values (Fig. 2), the DI class (Table 1) will not necessary be the same for them, even when simulated DI is close to real DI. This is mainly true to values close to the boundary limits between classes. For example, if simulated DI, using Fig. 1 equation, is equal 49 and the real DI value is 51 , they would be classified in different classes, moderately dry and sub-humid, respectively, according to Table 1 . So, the use of VWI instead of DI could not be generalized for MCC System classification purposes.

\section{Conclusion}

The excellent performance of the quadratic model shows that it could be possible to estimate the MCC System Dryness Index (DI) using the Vineyard Water Indicator (VWI) values.

\section{References}

[1] M.F. Cabré, H. Quénol, M. Nuñez, Int. J. Biom., 60, 1325-1340 (2016)

[2] H. Fraga, J.A. Santos, A.C. Malheiro, A.A. Oliveira, J. Moutinho-Pereira, G. V. Jones, Int. J. Clim., 36, 1-12 (2016)

[3] W.S. Ricce, S.L.C. Carvalho, P.H. Caramori, S.R. Roberto, Sem. C. Agr., 35, 2327-2336 (2014)

[4] A.C. Malheiro, J.A. Santos, H. Fraga, J.G. Pinto, Clim. Res., 43, 163-177 (2010)

[5] J. Tonietto, A. Carbonneau, Agr. For. Met., 124, 81-97 (2004)

[6] H. Fraga, A.C. Malheiro, J. Moutinho-Pereira, G.V. Jones, F. Alves, J.G. Pinto, J.A. Santos, Reg. Env. Chan., 14, 295-306 (2014)

[7] F.J. Moral, F.J. Rebollo, L.L. Paniagua, A. García, E.M. Salazar, Theo. Appl. Clim., 123, 277-289 (2016)

[8] A.H.C. Teixeira, J. Tonietto, G.A. Pereira, F. Angelotti, Rev. Bras. Eng. Agr. Amb., 16, 399-407 (2012)

[9] M.A.F. Conceição, J. Tonietto, F.B. Fialho, Ver. Bras. Frut., 34, 175-182 (2012)

[10] F.P. Gomes, An. Esc. Sup. Agr. L. Q., 355-368 (1959)

[11] A.P Camargo, P.C. Sentelhas, Rev. Bras. Agrom., 5, 89-97 (1997) 